\title{
Prehabilitación en pacientes oncológicos: Una revisión bibliográfica.
}

\section{Prehabilitation in cancer patients: A systematic review.}

Pág. 12,17

Recibido: 07-05-2020

Dra. Sofía Segreda Castro. Nutrición y Medicina General. San José, Costa Rica.

Aceptado: 13-05-2020

Dr. Alexander Muñoz Porras. Cirujano Oncólogo, Hospital San Juan de Dios, Costa Rica.

\section{Resumen:}

La prehabilitación constituye una novedosa opción terapéutica que brinda tanto a médicos como pacientes, los métodos y las herramientas necesarias para optimizar el estado físico, emocional y nutricional de los enfermos, en el periodo comprendido entre el diagnóstico y el inicio del tratamiento.

Como parte del proceso de prehabilitación en primera instancia, se debe de determinar el estado basal de cada enfermo, para poder establecer medidas individualizadas de acuerdo con las necesidades y deficiencias de cada uno. El objetivo de esta fase es mejorar la tolerancia a los tratamientos e intervenciones propuestas, así como facilitar la recuperación y reintegración de estos pacientes de forma temprana.

Palabras clave: Cáncer, prehabilitación, nutrición.

\section{Abstract:}

Prehabilitation is a new therapeutic option that provides physicians and patients with the necessary methods and tools to optimize physical, emotional and nutritional status in the period between diagnosis and the initial stage of treatment.

It is considered essential to first determine the baseline status of each patient in order to establish individualized measures according to the needs and deficiencies of each person. The objective of this phase is to improve tolerance to the proposed treatments and interventions, as well as to facilitate an earlier recovery and reintegration of these patients.

Key words: Cancer, prehabilitation, nutrition.

\section{Introducción:}

El cáncer es uno de los principales problemas de salud que enfrenta la sociedad en la actualidad. Durante el año 2015 se diagnosticaron alrededor de 17.5 millones de casos y fue la causa de muerte de aproximadamente 8.7 millones de personas a nivel global. De esta forma, el cáncer a nivel mundial se ha convertido en la segunda causa de defunción, siendo superado únicamente por las enfermedades cardiovasculares (1).

En los pacientes oncológicos, con gran frecuencia se identifican consecuencias físicas, psicológicas y sociales debidas a su diagnóstico y respectivo tratamiento, con severas repercusiones en los diferentes ámbitos de la vida (2).

Por este motivo se considera de suma importancia establecer estrategias que ayuden a mitigar los efectos adversos de las terapias en las vidas de los pacientes. Es así como surge el concepto de prehabilitación, o fase de pretratamiento, el cual propone alcanzar una adecuada preparación física y emocional previo al inicio de la fase terapéutica, con el objetivo de facilitar la recuperación, disminuyendo la utilización de servicios de salud ${ }^{2}$.

Actualmente la prehabilitación incorpora el concepto de mitigación o prevención del deterioro, la cual se puede aplicar a diferentes patologías, entre estas al cáncer (3).

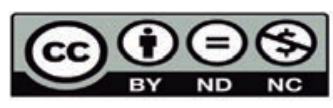


La prehabilitación se define como un proceso que mejora la capacidad funcional y mental de un individuo con el fin de amortiguar los posibles efectos nocivos ante un factor significativamente estresante (3). Utilizando previo al tratamiento, las intervenciones necesarias para preparar al paciente física y emocionalmente, y que así pueda sobrellevar con mayor éxito los impactos inmediatos y a largo plazo ocasionados por el procedimiento necesario para tratar su patología (4).

Se ha demostrado que el acondicionamiento psicológico y físico en los pacientes, antepuesto a la cirugía, ha mejorado las reservas tanto mentales como corporales para enfrentar de mejor manera la etapa postquirúrgica (3).

La fase de pre tratamiento se establece en el periodo de tiempo transcurrido desde el diagnóstico hasta el inicio del tratamiento e incluye asesorías psicológicas y físicas que en principio determinarán el nivel funcional basal, ayudarán a identificar discapacidades y a definir estrategias para intervenciones físicas y psicológicas (3).

Es muy importante enfatizar la diferencia entre prehabilitación y recuperación postquirúrgica mejorada (ERAS), esta última incluye elementos dirigidos a reducir el estrés perioperatorio, mantener una adecuada función fisiológica postquirúrgica y acelerar la recuperación luego de una cirugía (5). Mientras que la prehabilitación, como se explicó anteriormente, se enfoca en la mejora de la capacidad funcional y mental del paciente para enfrentar de manera más exitosa los tratamientos venideros (3) (4).

La presente revisión bibliográfica se enfocará únicamente en la prehabilitación de pacientes con diagnóstico de cáncer.

\section{Prehabilitación:}

Desde el punto de vista fisiológico, los procedimientos quirúrgicos ocasionan en los individuos un gran impacto en la homeostasis, llevando a un aumento en el consumo de oxígeno y así como un incremento en el catabolismo (6).

La respuesta del cuerpo humano ante la lesión consiste en una reacción sistémica orientada a mantener las funciones vitales y restaurar la homeostasis. La cirugía electiva se considera un factor estresante programado. Es por esto que la idea de prevenir o atenuar la respuesta ante el estrés quirúrgico ha sido sumamente atractiva durante los últimos años, es en este punto donde adquiere relevancia el identificar, especialmente, los individuos con capacidad funcional reducida, la cual se encuentra principalmente determinada por el estado nutricional, psicológico y el acondicionamiento físico, considerándose todos estos factores de riesgo modificables (7).

\section{Psicología:}

La depresión y la ansiedad son diagnósticos encontrados con frecuencia en pacientes con cáncer (8). Diversos estudios han logrado determinar que los trastornos psicológicos impactan negativamente tanto en el control del dolor, cicatrización de heridas como en el aumento en las estancias hospitalarias, de la misma forma que se asocian con disminución en la respuesta inmune y con aumento de la mortalidad (9).

Debido a esto se han propuesto varias terapias psicológicas para tratar estos padecimientos, entre ellas se encuentran las estrategias de resolución de problemas y afrontamiento, consejería y técnicas de relajación, entre otras. Tradicionalmente estas intervenciones se han aplicado luego del tratamiento del cáncer, no obstante, ahora se reconoce que el periodo perioperatorio es muy perturbador para estos enfermos, en este sentido, cada vez existe evidencia más robusta que apoya la psicoterapia previa a la intervención quirúrgica, ya que ha demostrado tener un impacto positivo en la recuperación post operatoria mejorando la calidad de vida de los pacientes (10).

Algunos estudios, han establecido que los tratamientos psicológicos previos a una cirugía 
logran disminuir los niveles de cortisol en el cuerpo humano y mejoran el proceso de cicatrización luego de una intervención abdominal (10).

Existe evidencia que apoya la terapia psicológica durante la fase de pretratamiento, ya que mejora la calidad de vida, disminuye la ansiedad y depresión, mejora el control del dolor y reduce la fatiga. Además, también se cree que estos tipos de terapias conllevan a una mejoría en el funcionamiento físico, la habilidad para deambular y para realizar tareas de rehabilitación (11).

\section{Ejercicio:}

El estado físico es un elemento determinante para la consecución de adecuados resultados posteriores a una cirugía, es por este motivo que es uno de los componentes que conforma las guías de evaluación cardiovascular pre operatoria para cirugías mayores no cardíacas del Colegio Americano de Cardiología/Asociación Americana del Corazón (12).

La prueba de ejercicio cardiopulmonar cada vez se reconoce más como el estándar de oro para evaluar el riesgo quirúrgico en personas que serán sometidas a un procedimiento mayor, debido a que esta prueba facilita la toma de decisiones en cuanto al manejo peri e intraoperatorio (12).

Diferentes revisiones sistemáticas han establecido de forma consistente la relación entre la condición física del paciente, definida por medio de la prueba de ejercicio cardiopulmonar, y los resultados posteriores al tratamiento quirúrgico. En esta misma línea, diversos estudios han demostrado que un menor consumo de oxígeno en el umbral del lactato está asociado con un aumento en la mortalidad post operatoria, por lo que este es otro factor importante en estos pacientes (12).

El cáncer y sus tratamientos se encuentran asociados con un declive en la condición física llevando a consecuencias negativas en la calidad de vida de las personas (7) (13). Los principales componentes del funcionamiento físico prequirúrgico, son la capacidad de resistencia aeróbica y la fuerza muscular (14), ambos elementos generalmente se encuentran disminuidos en estos pacientes, lo cual se establece como un factor predictor de complicaciones post operatorias y mayor riesgo de mortalidad (15).

En los últimos años, se han realizado diferentes estudios que evalúan la posibilidad de mejorar la función física previo al inicio del tratamiento, siendo parte fundamental de la prehabilitación como mecanismo para atenuar el estrés operatorio y así optimizar la recuperación post quirúrgica (15).

El acondicionamiento físico en la fase pre tratamiento consiste mayormente de ejercicios de resistencia para aumentar la masa muscular y la respuesta aeróbica y de fuerza, siendo este último el más efectivo durante dicho periodo, ya que es el que se ha asociado con mayor impacto positivo en la función cardiorrespiratoria (15).

Aunque la evidencia que apoya el uso de la preparación física para mejorar esta condición previo a que comience la fase de tratamiento hasta ahora ha sido escasa (15), cada vez existen más estudios que favorecen el ejercicio físico como parte esencial de la terapia en la prehabilitación (14).

Alguna de esta evidencia secunda que la capacidad funcional de los individuos es de extrema importancia para preservar la salud y que por tanto debe de optimizarse antes, después de la cirugía y durante cualquier tratamiento adicional, como lo suelen ser quimioterapia y/o radiación (14). De forma adicional, algunos estudios también señalan que la actividad física constituye un factor coadyuvante en el mejoramiento de la respuesta inmune del paciente (13).

Aún no existen protocolos específicos de ejercicio cardiovascular o resistencia en el periodo de prehabilitación en pacientes con cáncer. Sin embargo, a este respecto, la Sociedad Americana del Cáncer recientemente publicó sus guías, 
en las que sugieren realizar cada semana al menos 150 minutos de ejercicio con intensidad moderada o 75 minutos de ejercicio vigoroso o una combinación de ambos, además recomiendan incorporar entre 2 a 3 sesiones de entrenamiento de resistencia, incluyendo los grupos musculares más importantes (9).

Por otro lado, el entrenamiento se basa en el principio de sobrecarga, por lo tanto, a medida que el paciente se acostumbra a las demandas del ejercicio realizado, la intensidad deberá incrementarse progresivamente, siempre manteniendo un balance entre la intensidad/ cantidad ideal de ejercicio (11).

Las prescripciones de ejercicio idealmente deben ser adaptadas al nivel basal de la condición física de cada persona, al procedimiento quirúrgico planificado y al estado de salud actual. En otras palabras, tiene que adecuarse a las necesidades individuales de cada paciente. Es sumamente importante remarcar que en algunos individuos la actividad física estará completamente contraindicada o deberá modificarse en función de su estado de salud, ya que es conveniente mantener la seguridad en la población portadora de neoplasias (9).

\section{Nutrición:}

La malnutrición es prevalente en el $39 \%$ de las personas con cáncer (12), genera un gran impacto en la vida de los seres humanos y es una de las condiciones que continúan siendo infra tratadas en los pacientes oncológicos (7). Entre las causas de pérdida de peso y mala nutrición en pacientes con tumores se encuentra la obstrucción del tracto gastrointestinal (TGI) debida al tumor, anorexia inducida por la neoplasia, alteración en el metabolismo de nutrientes, disminución en la ingesta alimenticia luego del tratamiento contra su patología, efectos catabólicos del cáncer, ansiedad, depresión e inapetencia debida al dolor (12).

En cuanto a los factores independientes relacionados con la malnutrición en el cáncer se pueden enumerar los siguientes: nivel socioeconómico bajo, edad mayor o igual a 60 años, tabaquistas o ex tabaquistas y nivel de funcionamiento mayor o igual a 2 según la Clasificación Internacional del Funcionamiento, de la Discapacidad y de la Salud de la Organización Mundial de la Salud (OMS)) (12).

Se sabe que la subalimentación en neoplasias se encuentra asociada con mayor estancia hospitalaria, riesgo de complicaciones quirúrgicas, aumento de mortalidad y menos tolerancia a terapias adyuvantes (7).

Tanto el tumor en sí, como la cirugía para tratar este padecimiento predisponen a inflamación sistémica como respuesta del organismo y esto puede llevar a resistencia anabólica, alteraciones en insulina, gasto energético, lipólisis, proteólisis y excreción hormonal (7). Otro de los factores determinantes de la severidad en el déficit nutricional es la localización de la patología, siendo mayor en cánceres de tracto gastrointestinal, cabeza y cuello y menor en los de próstata y mama.

Además, esta deficiencia también dependerá de la terapia requerida por cada paciente, algunos reportes indican que durante el tratamiento $52.6 \%$ de los enfermos presentan desnutrición severa, $31.6 \%$ malnutrición moderada y solamente $15 \%$ tienen un adecuado nivel nutricional (12).

Previo a establecer cuáles serán las recomendaciones dietéticas es indispensable realizar la valoración nutricional del paciente oncológico y aunque no se reconoce ninguna herramienta como estándar de oro (12), tradicionalmente se han utilizado los niveles de albúmina en plasma, la pérdida de peso involuntaria, el índice de masa corporal (IMC) y el IMC ajustado a la pérdida ponderal no voluntaria, todos estos métodos continúan siendo de gran relevancia (7). Sin embargo, en la actualidad se han establecido nuevas metodologías como lo son la Valoración Global Subjetiva (VGS) y Valoración Global Subjetiva Generada por el Paciente (VGSGP), las que cada vez ocupan un papel más 
protagónico para determinar el estado nutricional en pacientes oncológicos (9).

La VGS clasifica la condición nutricional de los individuos hospitalizados, sean quirúrgicos o no, basándose en el examen físico (pérdida de grasa subcutánea, masa muscular y edema o ascitis) y en la historia clínica del individuo (pérdida de peso, capacidad funcional, cambios en la alimentación, síntomas gastrointestinales); mientras que la VGS-GP es una forma más detallada de la VGS, y aparte de determinar el estado nutricional, también establece el pronóstico y resultados post operatorios; esta última herramienta mencionada está indicada en pacientes que son admitidos en un centro hospitalario ${ }^{9}$.

El principal objetivo de la atención nutricional en los pacientes portadores de neoplasias es disminuir la incidencia y severidad de las náuseas y vómitos, ayudar a mantener la normoglicemia, aumentar el apetito, mejorar el sistema inmune, proveer suficiente energía para mantener el peso corporal y proteína para alcanzar el anabolismo ${ }^{9}$. Cada día existe más evidencia que apoya el uso de las intervenciones nutricionales de forma prequirúrgica, las cuales se deben basar en establecer la mala nutrición de forma pre operatoria para brindar tratamiento nutricional ajustado a las necesidades de cada paciente y así mejorar el estado metabólico, los resultados post operatorios y disminuir la necesidad de soporte nutricional posterior a la cirugía (9).

En cuanto al plan nutricional de estas personas deben tomarse en cuenta ciertas consideraciones explicadas a continuación:

A) Dieta hiperproteica: los requerimientos proteicos se encuentran elevados en estados de estrés, como las patologías oncológicas (11), gracias a la alteración en el metabolismo muscular de proteínas, por lo que una adecuada ingesta proteica es esencial en la fase de pretratamiento. Basándose en las guías internacionales, la ingesta proteica diaria en la prehabilitación debe ser 1.5 $\mathrm{g} / \mathrm{kg}$ de peso ideal. Las proteínas de alta calidad como las encontradas en los lácteos, vegetales, pescado, huevos y aves de corral, representan la fuente de primera línea; mientras que los suplementos nutricionales deberán indicarse únicamente cuando el paciente realmente lo requiera. Los aminoácidos esenciales como la leucina y la proteína de soya son importantes desencadenantes anabólicos y deben preferirse sobre otras fuentes de suplementación proteica. Los pacientes con enfermedad renal crónica moderada o avanzada y sin diálisis, no deben someterse a ingestas de proteínas mayores a 1 $\mathrm{g} / \mathrm{kg} / \mathrm{dí}^{7}$.

B) Dieta balanceada: Se debe garantizar la ingesta de proporciones adecuadas de todos los grupos de macronutrientes. La alimentación balanceada está compuesta por carbohidratos, proteínas (o sus fuentes alternativas) y lípidos en una relación 2:1:2 y los pacientes deberán recibir recomendaciones nutricionales generales y de una manera en que se les facilite seguirlas y entenderlas. Algunos ejemplos de sugerencias nutricionales son: reducir la ingesta de alcohol, limitar alimentos ricos en azúcares simples, restringir la ingesta de alimentos ricos en grasas, entre otros (7).

C) Requerimiento energético: El requerimiento de energía está compuesto por la cantidad de micro y macronutrientes necesarios para cubrir el gasto energético diario, promover procesos fisiológicos metabólicos y almacenar energía. En los pacientes oncológicos las necesidades de energía son muy variables y muchos de ellos muestran hipermetabolismo. El estado hipermetabólico se considera un marcador temprano de caquexia en pacientes con tumores y está relacionado con un balance energético negativo, inflamación sistémica y pérdida de peso. Esta condición generalmente ocurre en individuos con buen estado físico, por lo que su pronta detección es de suma importancia. Para el cálculo del gasto energético existe la fórmula de Harris Benedict, sin embargo, al ser un método muy poco preciso, se recomienda utilizar siempre que se pueda la calorimetría indirecta (7). 


\section{Conclusiones:}

El número de enfermos por cáncer ha aumentado en forma significativa en las últimas décadas y se prevé que este comportamiento epidemiológico se mantenga en los siguientes años. Esto asociado con el desarrollo de agresivos abordajes terapéuticos multidisciplinarios, ha generado un nuevo reto, el cual consiste en mejorar la tolerancia y respuesta de los enfermos en sus tratamientos.

La prehabilitación ha demostrado, hasta ahora, que ofrece una ventana de oportunidad para mejorar el estado fisiológico y psicológico de los enfermos. Ha quedado en evidencia que los pacientes a quienes se les ofrece la fase de pretratamiento, tienen estancias hospitalarias y periodos de recuperación reducidos, así como una más pronta reintegración a las actividades ordinarias previas a la enfermedad.

Se considera que al día de hoy deben realizarse más estudios de mayor escala que permitan establecer cuáles son las personas que obtendrían mayor beneficio de la prehabilitación, así como cuáles son las medidas que ofrecen mejor relación costo - beneficio para los sistemas de salud.

\section{Bibliografía:}

1.Fitzmaurice C. Global, regional, and national cancer incidence, mortality, years of life lost, years lived with disability, and disability-adjusted life-years for 32 cancer groups, 1990 to 2015: a systematic analysis for the global Burden of disease study. HHS Public Access. 2017 Abril ; 3(4).

2.Treanor CKTDM. An international review and metaanalysis of prehabilitation compared to usualcare for cancer patients. Springer. 2017 Setiembre; 12.

3.Santa DSBCGCCF. Optimizacion of Surgical outcomes with prehabilitation. NRC Research Press. 2015 Mayo; 40(9).

4.Gillis CFTSTMEARLSea. Trimodal prehabilitation for colorectal surgery attenuates post-surgical losses in lean body mass: a pooled analysis of randomized controlled trials. Clinical Nutrition. 2019 Junio; 38(3).

5.Gustafsson U. Scott MHMNJDNFNea. Guidelines for perioperative care in elective colorectal surgery: Enhaced Recovery After Surgery (ERAS) society recommendations: 2018. World Journal of Surgery. 2018 Noviembre; 43.

6.Wynter-Blyth VMK. Prehabilitation: preparing patients for surgery. The BMJ. 2017 Agosto.

7.Minnella ECF. Prehabilitation and functional recovery for colorectal cancer patients. European Journal of Surgical Oncology. Julio 2018; 44(7).

\section{Scheede-Bergdahl CMECF. Multi-modal} prehabilitation: addressing the why, when, what, how, who and where next? Association of Anaesthetists.
2019; 74(1).

9.Carli FSJFLMAGSGCea. Surgical prehabilitation in patients with cancer. State-of-the-science and recommendations for future research from a panel of subject matter experts. Elsevier. 2017 Febrero; 28(1).

10.Tsimopoulou IPSHRDAGDTlea. Psychological prehabilitation before cancer surgery: a systematic review. Annals of Surgical Oncology. 2015 Abril ; 22.

11. Carli FGCSBC. Promoting a culture of prehabilitation for the surgical cancer patient. Acta Oncológica. 2017 Enero; 56(2).

12.LoughneyLGM. Exercise and nutrition prehabilitation for the evaluation of risk and therapeutic potential in cancer patients: a review. International Anesthesiology Clinics. 2016; 54(4).

13.Onerup AAEBDBMOMGEea. The effect of preand post-operative physical activity on recovery after colorectal cancer surgery (PHYSSURG-C): study protocol for a randomised controlled trial. Biomed Central. 2017 Mayo; 18.

14.Van Rooijen SEMSBCCFRRSGea. Systematic review of exercise training in colorectal cancer patients during treatment. Scandinavian Journal of Medicine and Science in Sports. 2017 Mayo; 28(2).

15.Piraux ECGRG. Effects of preoperative combined aerobic and resistance exercise training in cancer patients undergoing tumour resection surgery: a systematic review of randomised trials. Elsevier. 2018 Setiembre; 27(3). 